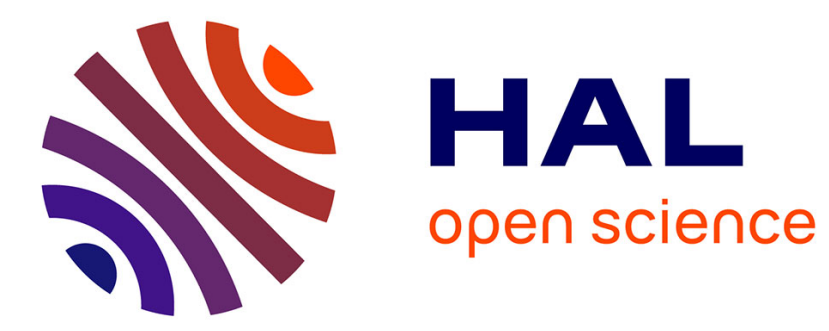

\title{
Inverse Optimal Control to Model Human Trajectories During Locomotion
}

\author{
Isabelle Maroger, Olivier Stasse, Bruno Watier
}

\section{To cite this version:}

Isabelle Maroger, Olivier Stasse, Bruno Watier. Inverse Optimal Control to Model Human Trajectories During Locomotion. Computer Methods in Biomechanics and Biomedical Engineering, 2022, 25 (5), pp.499-511. 10.1080/10255842.2021.1962311 . hal-03142655v2

\section{HAL Id: hal-03142655 \\ https://hal.laas.fr/hal-03142655v2}

Submitted on 24 Aug 2021

HAL is a multi-disciplinary open access archive for the deposit and dissemination of scientific research documents, whether they are published or not. The documents may come from teaching and research institutions in France or abroad, or from public or private research centers.
L'archive ouverte pluridisciplinaire HAL, est destinée au dépôt et à la diffusion de documents scientifiques de niveau recherche, publiés ou non, émanant des établissements d'enseignement et de recherche français ou étrangers, des laboratoires publics ou privés. 


\title{
RESEARCH ARTICLE
}

\section{Inverse Optimal Control to Model Human Trajectories During Locomotion}

\author{
Isabelle Maroger ${ }^{\mathrm{a}}$ and Olivier Stasse ${ }^{\mathrm{a}}$ and Bruno Watier ${ }^{\mathrm{a}}$ \\ aLAAS-CNRS, Université de Toulouse, CNRS, UPS, France
}

\section{ARTICLE HISTORY}

Compiled August 24, 2021

\begin{abstract}
Cobotic applications require a good knowledge of human behaviour in order to be cleverly, securely and fluidly performed. For example, to make a human and a humanoid robot perform a co-navigation or a co-manipulation task, a model of human walking trajectories is essential to make the robot follow or even anticipate the human movements. This paper aims to study the Center of Mass (CoM) path during locomotion and generate human-like trajectories with an optimal control scheme. It also proposes a metric which allows to assess this model compared to the human behaviour. CoM trajectories during locomotion of 10 healthy subjects were recorded and analysed as part of this study. Inverse optimal control was used to find the optimal cost function which best fits the model to the measurements. Then, the measurements and the generated data were compared in order to assess the performance of the presented model. Even if the experiments show a great variability in human behaviours, the model presented in this study gives an accurate approximation of the average human walking trajectories. Furthermore, this model gives an approximation of human locomotion good enough to improve cobotic tasks allowing a humanoid robot to anticipate the human behaviour.
\end{abstract}

\section{KEYWORDS}

locomotion analysis; modeling; optimal control; model-based simulation; human-robot interaction 


\section{Nomenclature}

CoM: Center of Mass

OC: Optimal Control

IOC: Inverse Optimal Control

$(x, y)$ : horizontal CoM position

$\gamma, \theta$ : orientation of the pelvis in the local and global frames

$v_{\text {forw }}$ : forward velocity

$v_{\text {orth }}$ : orthogonal velocity

$X$ : state variable of the OC problem

$X_{0}, X_{f}$ : initial and goal states

$U$ : control variable of the $\mathrm{OC}$ problem

$T$ : duration of the travel

$\phi_{r}, \phi_{t}$ : running and terminal cost functions of the OC problem $\alpha, \beta$ : weights of the running and terminal costs

$X^{m e s}=\left(\begin{array}{lll}X_{1}^{m e s} & \ldots & X_{N}^{m e s}\end{array}\right)^{T}$ : measured trajectory for one subject

$\bar{X}^{m e s}=\left(\begin{array}{lll}\bar{X}_{1}^{m e s} & \ldots & \bar{X}_{N}^{m e s}\end{array}\right)^{T}$ : average measured trajectory for all subjects without distinction $X^{g e n}=\left(\begin{array}{lll}X_{1}^{g e n} & \ldots & X_{N}^{g e n}\end{array}\right)^{T}$ : generated trajectory

$N$ : number of points in the trajectories $d$ : global distance

$d_{x y}, d_{\gamma}$ : linear and angular distances

$\bar{d}_{x y}, \bar{d}_{\gamma}$ : linear and angular errors

$D$ : cost function of the IOC problem

\section{Introduction}

Every interaction between a humanoid robot and a human is a great challenge as humanoid robots are very complex systems due to their numerous degrees of freedom and their natural instability. These interactions go from avoiding the humans to assisting them when doing complex tasks. In the context of collaborative tasks, humanoid robots classically follow passively the humans (Kosuge et al. 1993). Nevertheless, those tasks could be performed more efficiently if the robot can predict and anticipate the human motions. Making a robot actively cooperate with a human requires a good knowledge of the human behaviour. Thus, in the context of the French ANR-COBOT project, dedicated to a collaborative table handling task between a human and a humanoid robot, models of human walking trajectories and forces applied by the human on the table are needed to allow the robot to real-time assist the human. This work, conducted as part of the ANR-COBOT, focuses on the first step of this co-manipulation task, namely reaching the table. This study aims to model and generate human trajectories during locomotion to make the robot walk to the table in a human-like way. The way humans walk with a table or interact with a robot to handle a table will be handled in further works. Nevertheless, the method is aimed to be the same. Moreover, evidences of better human-robot interactions under various forms of humans control were already demonstrated (Sheridan 2016). This is why we hypothesise that an accurate understanding and model of the human locomotion will help the human partner to understand where the robot endpoint is.

\subsection{State of the Art}

In biomechanics, numerous authors study and describe healthy human gait (Kim and Bertram 2018; Bovi et al. 2011) . Some authors are interested in human walking trajectories, especially during collision or obstacles avoidance (Basili et al. 2013; Heijnen et al. 2012) while others studied and modelled the human joint trajectories during gait (Nandi et al. 2016, Semwal et al. 2018). Some more recent studies 
focus on the trajectories of human walking in crowded environment (Koilias et al. 2020: Zhu et al. 2020). However, to the best of our knowledge, the problem of the modeling and the simulation of common human trajectories during locomotion is not frequently raised. On the opposite, the generation of a path between a starting and a goal position is a commonly tackled problem in robotics. For mobile wheeled robot, numerous ways exist to generate paths. The easiest one is following a straight line while more complicated ones include B-splines (Elbanhawi et al. 2015) or curves generated with an Optimal Control (OC) model called the unicycle model (Soueres and Laumond 1996; Laumond 1998). For humanoid robots, some solutions use parametric curves called clothoids (Raković et al. 2019), as clothoid arcs have been showed to fit well human behaviour (Arechavaleta et al. 2008), or curves generated with an OC model based on Inverse Optimal Control (IOC) (Mombaur et al. 2010).

First of all, the unicycle model and the clothoid based model are non-holonomic locomotion models. This kind of model describes the locomotion of systems, called non-holonomic systems, which always move forward, never sideward, with their direction always tangent to their trajectory. The human can be approximated by a non-holonomic system when walking a straight line (Arechavaleta et al. 2001). This is why those models can be used to generate human walking paths (Papadopoulos et al. 2013; Arechavaleta et al. 2008). However, humans can also take sideward or oblique steps to avoid obstacles or go to close goals as explained in Mombaur et al. (2010). Those sideward motions are not taken into account by non-holonomic models. Thus, holonomic locomotion models allow more degrees of freedom in the human locomotion, like the one described in Mombaur et al. (2010), and should better describe human behaviour. Moreover, in Maroger et al. (2020b) and Maroger et al. (2020a), we already showed that an OC model adapted from Mombaur et al. (2010) better fits human trajectories than a clothoid based model according to a metrics which evaluates the distance between generated trajectories and measurements on human subjects. Both models aimed to generate smooth CoM trajectories. That is to say these trajectories did not take into account the medio-lateral oscillations due to the steps.

\subsection{Contribution}

The motivation of this research is to improve the simulation of human trajectories during locomotion compared to the previous papers published on this topic with more modalities of distance and orientation. To achieve this goal, a novel IOC scheme was used to find a suited cost function which allows the OC model, introduced in this paper, to generate trajectories which accurately fit the 400 experimental human trajectories measured as part of this study. The developed model aims to compute both the position and the orientation of a human-like system between any starting positions and a goal position. In order to assess this new OC model, a metrics was developed to measure the distance between the measured and the generated trajectories. The whole framework developed in this paper is shown in Fig.1. The model implementation uses open-source softwares with the aim to ease reproducibility. We hypothesise that this new model will be more efficient and accurate than the previous ones according to the defined metrics. We believe this model will allow to better predict human behaviour and improve humanoid robot-human cooperation. 


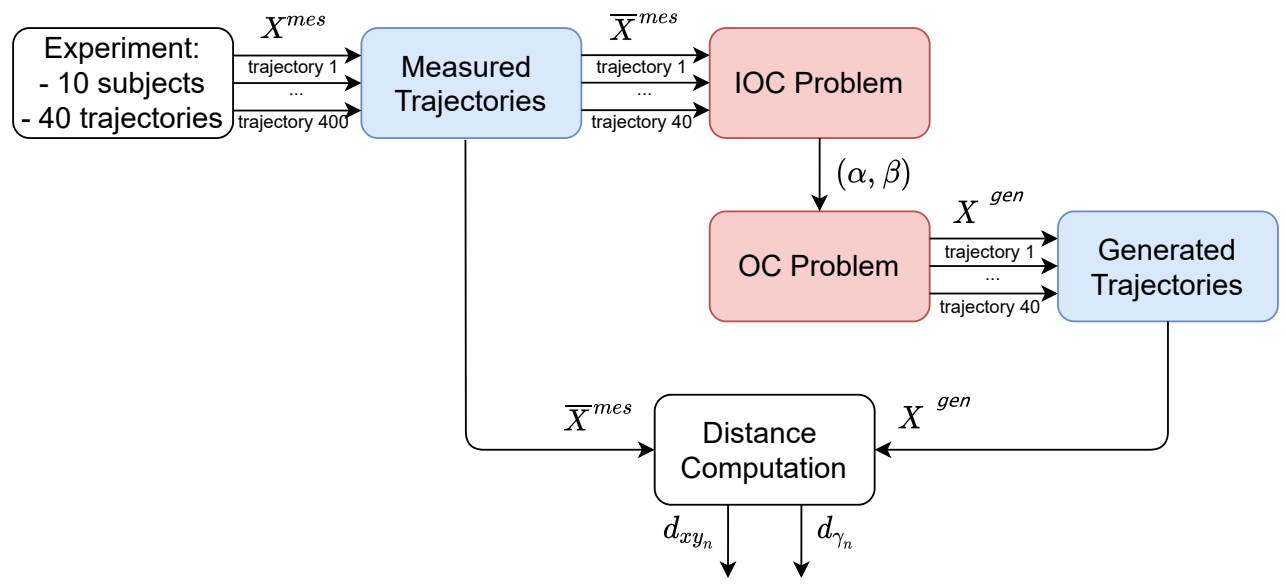

Figure 1. Description of the whole framework presented in the paper. The notations introduced in this chart are defined in the nomenclature and throughout the document.

\section{Methods}

\subsection{Participants}

Ten subjects ( 8 males and 2 females) volunteered for this study with a mean $( \pm$ standard deviation) age of $23.30 \pm 2.32$ years, height of $1.77 \pm 0.06 \mathrm{~m}$ and mass of $73.90 \pm 12.59 \mathrm{~kg}$. They all have no pathological disorders or medical conditions likely to alter their gait. Every participant was informed of the experimental procedure and gave its written consent before the experiment. However, they were not informed of the expected results in order to preserve their natural behaviour. This study was conducted in accordance with the declaration of Helsinki and with the approval of the University of Toulouse ethical committee.

\subsection{Experimental protocol}

The participants were instructed to walk from 10 starting positions with 4 different starting orientations $\left(-\frac{\pi}{2} \mathrm{rad}, 0 \mathrm{rad}, \frac{\pi}{2} \mathrm{rad}, \pi \mathrm{rad}\right)$ to one goal position placed in front of a table always with the same orientation $\left(\frac{\pi}{2} \mathrm{rad}\right)$. These positions are represented on Fig 2a. The subjects were asked to freely walk at a self selected normal speed. The starting positions were chosen to measure a set of typical locomotion path within a range of 0.6 to $5.5 \mathrm{~m}$ from the goal. The distance between the starting position and the goal position is denoted $d$ and called global distance in what follows.

In order to record the kinematics data of the subjects during locomotion, four passive markers were fixed on their pelvis. The $3 \mathrm{D}$ positions of these markers were collected thanks to a motion capture system (15 infrared VICON cameras sampling at $200 \mathrm{~Hz}$ ). Then, the horizontal CoM position $(x, y)$ was approximated by the middle of the two markers placed on the postero-superior iliac spine (Gard et al. 2004) and the orientation of the pelvis $\gamma$ was computed as the angle between the vector tangent to the trajectory and the cross product of the vector constructed with the two markers 


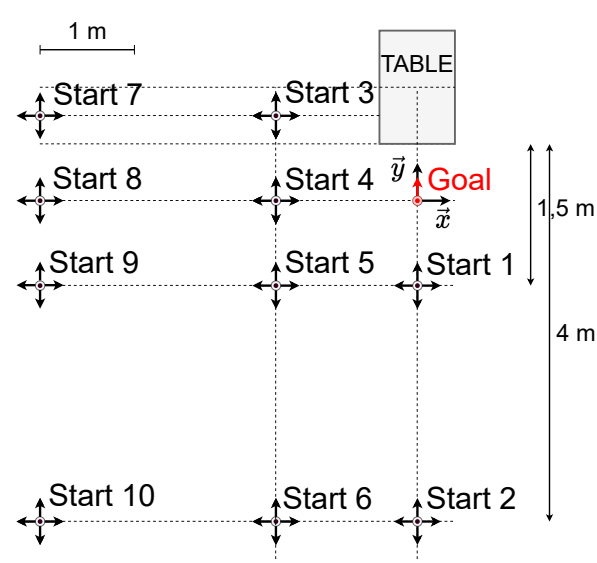

(a) Representation of the 10 starting positions and the goal position with their orientations.

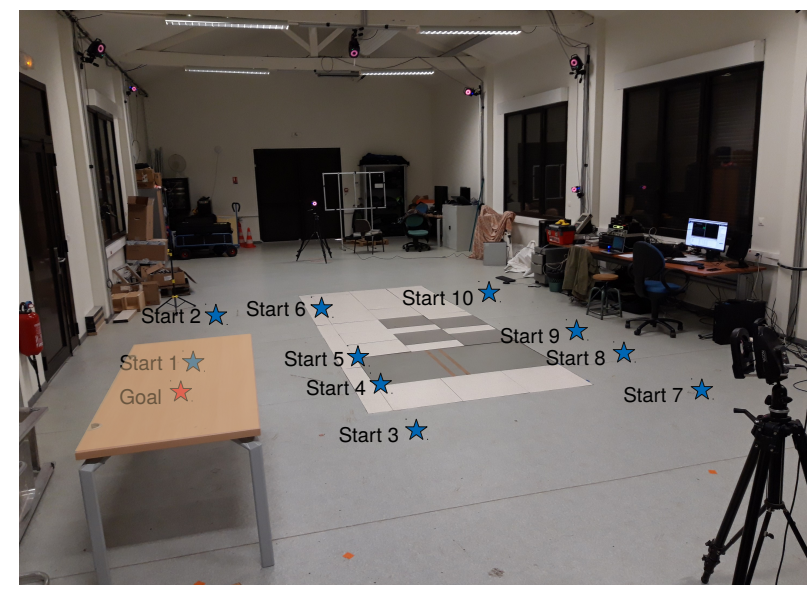

(b) Laboratory configuration.

Figure 2. Experimental setup

placed on the antero-superior iliac spine and the vertical direction. The laboratory configuration is represented in Fig 2b.

Kinematics data were filtered with a 4th order, zero phase-shift, low-pass butterworth with a $10 \mathrm{~Hz}$ cutoff frequency.

\subsection{Model description}

To simulate CoM trajectory during the locomotion in the horizontal plane, we used an OC scheme similar to the one proposed in Mombaur et al. (2010). The novelty of our work lies in the fact that we adapted the OC model presented in Mombaur et al. (2010) in order to solve it with a multiple shooting method with no strict equality final constraint. More precisely, we used a Differential Dynamic Programming (DDP) solver (Tassa et al. 2014) from the Crocoddyl library (Mastalli et al. 2020). This modification implied changes in the problem formulation, especially in some constraints which can no longer be strict equality constraints. This solver was chosen because it is open-source, which eases the reproducibility of this work, and is also efficient to solve OC problems for real time applications (Tassa et al. 2014).

As in Mombaur et al. (2010), a human subject was considered as a full holonomic locomotor system with the following dynamics:

$$
\left\{\begin{array}{l}
\dot{x}=\cos \theta \cdot v_{\text {forw }}-\sin \theta \cdot v_{\text {orth }} \\
\dot{y}=\sin \theta \cdot v_{\text {forw }}+\cos \theta \cdot v_{\text {orth }} \\
\dot{\theta}=\omega \\
\dot{v}_{\text {forw }}=u_{1} \\
\dot{v}_{\text {orth }}=u_{2} \\
\dot{\omega}=u_{3}
\end{array}\right.
$$

$(x, y)$ is the position of the human CoM in the horizontal plane. $\theta$ is the posterioanterior orientation of his pelvis in a global frame. $v_{\text {forw }}$ and $v_{\text {orth }}$ are the forward 


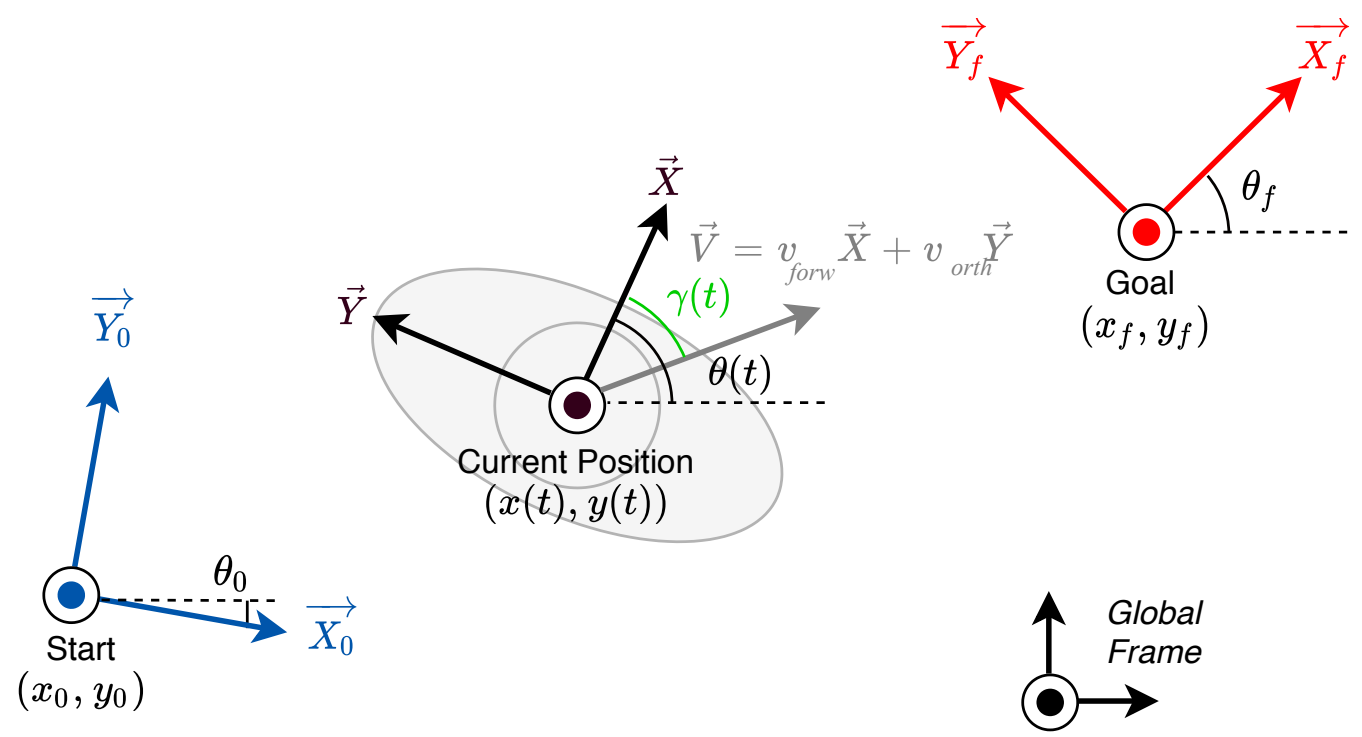

Figure 3. Coordinate systems in the trajectory problem to solve

and sideward velocities of the subject CoM in the local frame, in other words they are the tangent and orthogonal velocities with respect to the orientation of the pelvis. $\omega$ is his angular velocity. All the frames and variables involved here are represented on Fig. 3. In what follows, $u_{1}, u_{2}$ and $u_{3}$ are respectively named the forward, the orthogonal and the angular accelerations. As part of the formulation of the OC model $X=\left(x, y, \theta, v_{\text {forw }}, v_{\text {orth }}, \omega\right)^{T}$ is called the state and $U=\left(u_{1}, u_{2}, u_{3}\right)^{T}$ is called the control. The aim of the OC problem is to optimize the state, from which can be deduced the position $(x, y)$ and the orientation $\theta$ of the CoM, along a trajectory between a start and a goal positions. This dynamics does not take into account the medio-lateral oscillations due to the steps. It only allows to generate smooth CoM trajectories (Maroger et al. 2020b).

Then, we considered an OC model of the following form:

$$
\min _{X(.), U(.), T} \int_{0}^{T} \phi_{r}(X(t), U(t)) \mathrm{d} t+\phi_{t}(X(T))
$$

with $\phi_{r}$ and $\phi_{t}$ the running and terminal cost functions. $T$ is the time needed to go from the starting position to the goal position. This problem was solved under the following constraints:

$$
\begin{cases}\dot{X}=f(t, X(t), U(t)) & \text { Dynamical constraint (Eq } 1) \\ X(0)=X_{0} & \text { Initial constraint }\end{cases}
$$

with $X_{0}=\left(x_{0}, y_{0}, \theta_{0}\right)$ the initial state. 
$\phi_{r}$ and $\phi_{t}$ were chosen as follows:

$$
\left\{\begin{array}{c}
\phi_{r}(X(t), U(t))=\alpha_{0}+\alpha_{1} u_{1}^{2}(t)+\alpha_{2} u_{2}^{2}(t)+\alpha_{3} u_{3}^{2}(t) \\
+\alpha_{4} \psi\left(X(t), X_{f}\right)^{2} \\
\phi_{t}(X(T), U(T))=\beta_{0}\left(\left(x_{f}-x(T)\right)^{2}+\left(y_{f}-y(T)\right)^{2}\right) \\
+\beta_{1}\left(\theta_{f}-\theta(T)\right)^{2}+\beta_{2}\left(v_{\text {forw }}(T)^{2}+v_{\text {orth }}(T)^{2}\right)+\beta_{3} \omega(T)^{2}
\end{array}\right.
$$

$X_{f}=\left(x_{f}, y_{f}, \theta_{f}\right)$ is the goal state. $\alpha=\left(\alpha_{0}, \alpha_{1}, \alpha_{2}, \alpha_{3}, \alpha_{4}\right)$ are the weights of the running cost and $\beta=\left(\beta_{0}, \beta_{1}, \beta_{2}, \beta_{3}\right)$ the weights of the terminal cost. The function $\psi\left(X(t), X_{f}\right)=\arctan \frac{y_{f}-y(t)}{x_{f}-x(t)}-\theta(t)$ is the difference between the current orientation of the system and the angular difference between the orientation of the system and of its target. Thanks to this $\psi$ function, there is no symmetry between back and forth trajectories. This is representative of human locomotion as humans usually do not take the same path going from a start to a goal and from this goal to this start (Mombaur et al. 2010).

The function $\phi_{r}$ was already proposed to simulate CoM locomotion path (Mombaur et al. 2010). Moreover, $\phi_{t}$ was added here to act as a final constraint which imposes the system to reach the goal position and orientation with a zero velocity. In Mombaur et al. (2010), the weights $\alpha$ were set to $(1,1.2,0.7,1.7,5.2)$ using IOC. A similar method, described in Sec 2.5. was used to compute the weights $\alpha$ and $\beta$ of the cost functions in order to make the model best fit to the human behaviour. However, first, a metric needed to be defined to assess if the model generates trajectories which demonstrate a behaviour close to the measurements (Sec. 2.4).

Moreover, as stated in the Eq2, $T$ was to be optimized. However, the DDP solver only optimizes the state and the control. Thus, the OC problem must be solved with the DDP solver for different $T$ in order to find the best $T$ which minimizes the cost function. As $T$ represents the duration of the path between the start and the goal, we assumed that $T \geq \frac{d}{v}$ with $d=\sqrt{\left(x_{f}-x_{0}\right)^{2}+\left(y_{f}-y_{0}\right)^{2}}$ and $v=0.1 \mathrm{~m} / \mathrm{s}$. The limit velocity $v$ was chosen in accordance with the actual velocity displacement of the humanoid robot TALOS build by PAL Robotics (Stasse et al. 2017). Thus, the optimization of $T$ was done with the Nelder-Mead method of the Scipy library (Virtanen et al. 2020).

\subsection{Comparison between generated and measured trajectories}

The goal of this study is to design a model which generates trajectories as close from CoM human trajectories as possible. Thus, we needed to define a way to assess the closeness between the experimental and the generated trajectories, for example a distance between curves. This is why, we defined a distance between measured and generated trajectories similar to the one proposed in Arechavaleta et al. (2008). To this end, all the trajectories were normalized from 1 to $100 \%$ with a step of 0.1 . Then, for every of the 40 possible paths, from a starting position with one of the 4 orientations to the goal, a reference human trajectory was defined as the mean of the measured trajectories: $\bar{X}_{i}^{\text {mes }}=\frac{1}{10} \sum_{j=1}^{10} X_{i, j}^{\text {mes }}$ where $X_{i, j}^{\text {mes }}=\left(x_{i, j}^{\text {mes }}, y_{i, j}^{\text {mes }}, \gamma_{i, j}^{\text {mes }}\right)$ with $j \in[1,10]$ standing for the $j^{t h}$ subject and $i \in[1, N]$ with $N=1000$ for the $i^{\text {th }}$ point along the trajectory. This trajectory is called the average human trajectory. 
Now, the following distances can be computed:

$$
\left\{\begin{array}{l}
d_{x y}=\frac{1}{N} \sum_{i=1}^{N} \sqrt{\left(\bar{x}_{i}^{m e s}-x_{i}^{g e n}\right)^{2}+\left(\bar{y}_{i}^{m e s}-y_{i}^{g e n}\right)^{2}} \\
d_{\gamma}=\frac{1}{N-1} \sum_{i=1}^{N-1} \sqrt{\left(\bar{\gamma}_{i}^{m e s}-\gamma_{i}^{g e n}\right)^{2}}
\end{array}\right.
$$

$\left(\bar{x}^{\text {mes }}, \bar{y}^{\text {mes }}\right)$ is the average human trajectory over one path and $\left(x^{g e n}, y^{g e n}\right)$ is the corresponding generated trajectory. $\bar{\gamma}^{\text {mes }}$ is the average human orientation over one path and $\gamma^{g e n}$ corresponds to the angle between the vector tangent to the trajectory and the forward direction computed from the $\theta$ generated with the OC model as follows:

$$
\gamma_{i+1}=\arctan \frac{y_{i+1}-y_{i}}{x_{i+1}-x_{i}}-\theta_{i}
$$

In concrete terms, $d_{x y}$ represents the mean distance between two trajectories and $d_{\gamma}$ the mean angle between the experimental and the generated pelvis orientation. In the rest of the article, $d_{x y}$ and $d_{\gamma}$ are, respectively, called linear and angular distances.

To assess a trajectory generated with the OC model, we stated that the best generated trajectory minimizes the distances $d_{x y}$ and $d_{\gamma}$. Furthermore, we hypothesized here that the best model is the one which lower the mean distances over all the paths, namely $\bar{d}_{x y}=\frac{1}{40} \sum_{n=1}^{40} d_{x y_{n}}$ and $\bar{d}_{\gamma}=\frac{1}{40} \sum_{n=1}^{40} d_{\gamma_{n}}$. Those mean distances are called linear and angular errors.

Moreover, statistical tests needed to be performed in order to determine if the model is sensitive to the initial orientation or to the global distance. Thus, ANOVA (ANalysis Of VAriance) tests were performed to detect the influence of those criteria on the errors defined above $(p<0.05)$. Those tests were performed after checking the normality of the data with a Kolmogorov-Smirnov test. Mann-Whitney tests were also conducted to know if the distances between the generated trajectories and the average human trajectories and the distances between the measured trajectories for every subjects and the average human trajectories are significantly different $(p<0.05)$. Furthermore, a Kruskal test was performed to assess the variability of the chosen trajectory between the 10 subjects $(p<0.05)$.

\subsection{Inverse Optimal Control}

In Mombaur et al. (2009), the authors presented an inverse OC scheme based on the optimality criteria of human locomotion. They captured human walking paths to identify the weights of their OC cost function. This is called IOC. In this study, we applied the same method in order to find the weights $\alpha$ and $\beta$ of the cost functions defined in Eq.4.

IOC problems aim to determine the optimization parameters, here the weights of the cost functions, so that they make the solutions of the corresponding OC problem $\left(X^{g e n}, U^{g e n}\right)$ best fit the measurements $\left(\bar{X}^{\text {mes }}, \bar{U}^{\text {mes }}\right)$. As mentioned previously, the best fitting method minimizes the defined errors. Thus, the IOC problem can be defined 


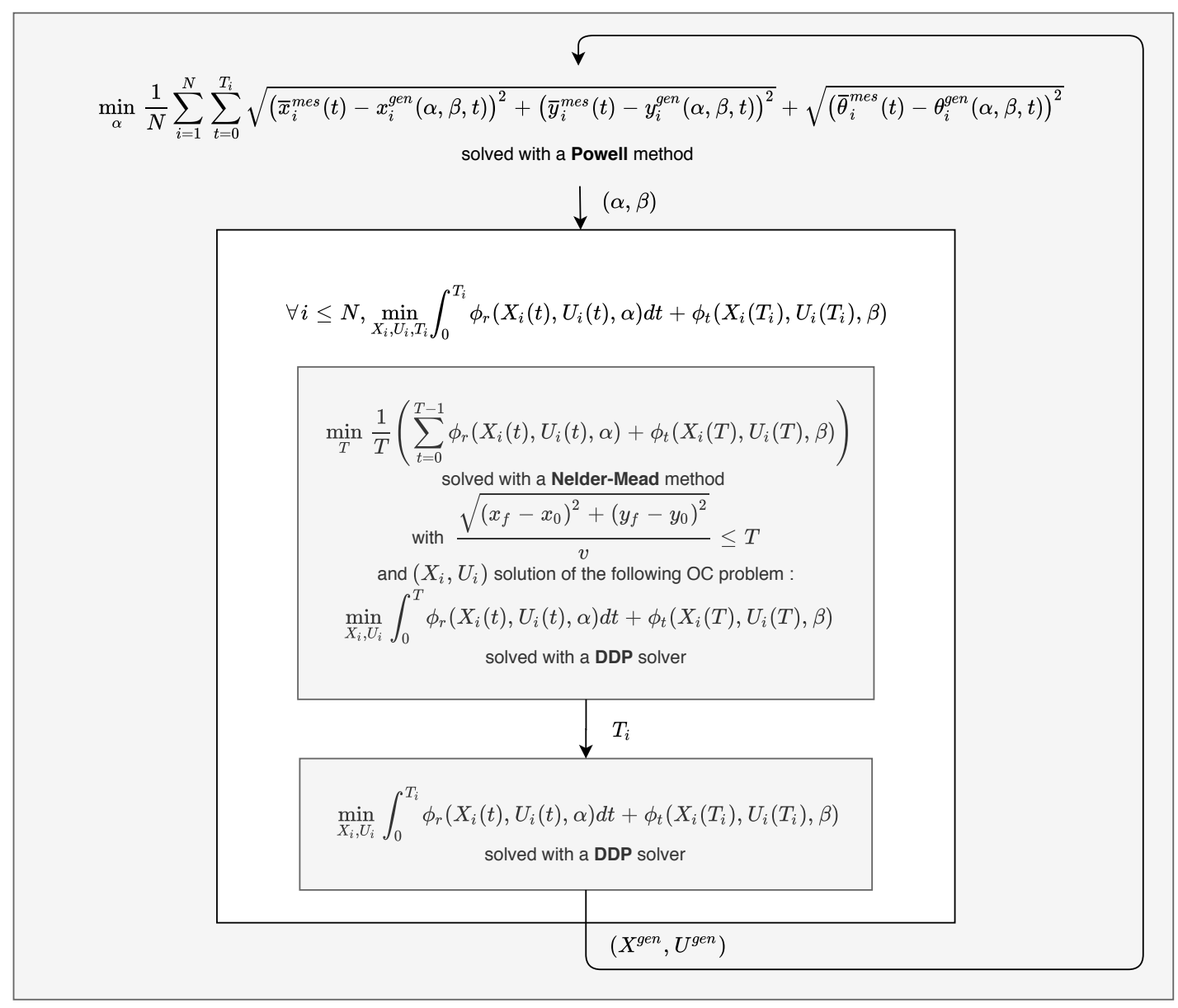

Figure 4. Algorithm to solve our IOC problem

as follows:

$$
\min _{\alpha, \beta} \frac{1}{40} \sum_{n=1}^{40} D\left(\bar{X}_{n}^{m e s}, X_{n}^{g e n}(\alpha, \beta)\right)
$$

with $D$ the cost function associated with this problem such as:

$$
D=d_{x y_{n}}\left(\bar{X}_{n}^{m e s}, X_{n}^{g e n}(\alpha, \beta)\right)+\frac{1}{2} d_{\gamma_{n}}\left(\bar{X}_{n}^{m e s}, X_{n}^{g e n}(\alpha, \beta)\right)
$$

This sum is weighted in order to have $d_{x y_{n}}$ and $d_{\gamma_{n}}$ of the same magnitude.

This kind of problem can be solved with a derivative free method like the Powell method (Powell 1964) of the Scipy library (Virtanen et al. 2020). The IOC problem that we solved in this paper is represented in Fig 4 . 


\section{Results}

\subsection{Human data analysis}

The mean and standard deviation of the linear and angular distances between the individual measurements and the average human trajectories are $0.1073 \pm 0.06519 \mathrm{~m}$ and $0.4842 \pm 0.1788 \mathrm{rad}$. Moreover, the measurements present a great variability according to the subjects as one can see on Fig.5. A Kruskal test demonstrates significant differences between the subjects for the linear distance $\left(p=3.09 \times 10^{-7}\right)$ and for the angular distance $\left(p=2.54 \times 10^{-6}\right)$. However, some subjects are not statistically distinguishable. Indeed, when performing a Kruskal test on 6 over the 10 subjects the obtained p-value is 0.31 for the linear distance and 0.34 for the angular distance. To conclude, some subjects have a similar behaviour while the others are totally distinguishable.

The box and whisker plots for linear and angular distances between the measurements and the average human trajectories are represented on Fig.7a (on the right). These plots show the median, the lower and upper quartile values $\left(Q_{1}\right.$ and $\left.Q_{3}\right)$ and extend from the maximum to the minimum within $\left[Q_{1}-1.5\left(Q_{3}-Q_{1}\right), Q_{3}+1.5\left(Q_{3}-Q_{1}\right)\right]$. The other values are considered as outliers.

\subsection{IOC results}

The weights of the cost function presented in Eq4 were optimized with the IOC problem described in Sec 2.5. Thus, the weights allowing the best fitting to human trajectories according to our metrics and our model are the followings:

$$
\left\{\begin{array}{l}
\left(\alpha_{0}, \alpha_{1}, \alpha_{2}, \alpha_{3}, \alpha_{4}\right) \approx\left(7.87,4.00,20.15,1.00 \times 10^{-6}, 10.00\right) \\
\left(\beta_{0}, \beta_{1}, \beta_{2}, \beta_{3}\right) \approx(10.00,10.00,0.38,3.36)
\end{array}\right.
$$

\subsection{Distance computation}

With these optimal weights, 40 trajectories corresponding to the same starts and goal than the human trajectories were generated using the OC model in order to evaluate this model. The average computational time of these generations scored to $1.45 \mathrm{~s}$. Four examples of these generated curves, one for each starting orientation, are shown in Fig.5.

The linear and angular distances were computed for each 40 generated trajectories. The obtained results for each starting positions and orientations are plotted on Fig 6 . Let us denote a moderate positive correlation between the mean linear distance and the global distance (with a Pearson correlation coefficient equal to 0.63) whereas there is a strong negative correlation between the mean angular distance and the global distance (with a Pearson correlation coefficient equal to -0.81).

Moreover, the computed errors ( \pm standard deviation) are $\bar{d}_{x y}=0.0767 \pm 0.0450 \mathrm{~m}$ and $\bar{d}_{\gamma}=0.3786 \pm 0.1336 \mathrm{rad}$. Thus, the results of the described OC model are close to the average human behaviour. However, the Mann-Whitney test comparing these errors and the distance between the measured trajectories and the average human trajectories demonstrates there is significant differences between the two data sets $\left(p=6.19 \times 10^{-4}\right.$ for the linear error and $p=3.56 \times 10^{-4}$ for the angular error $)$. On 

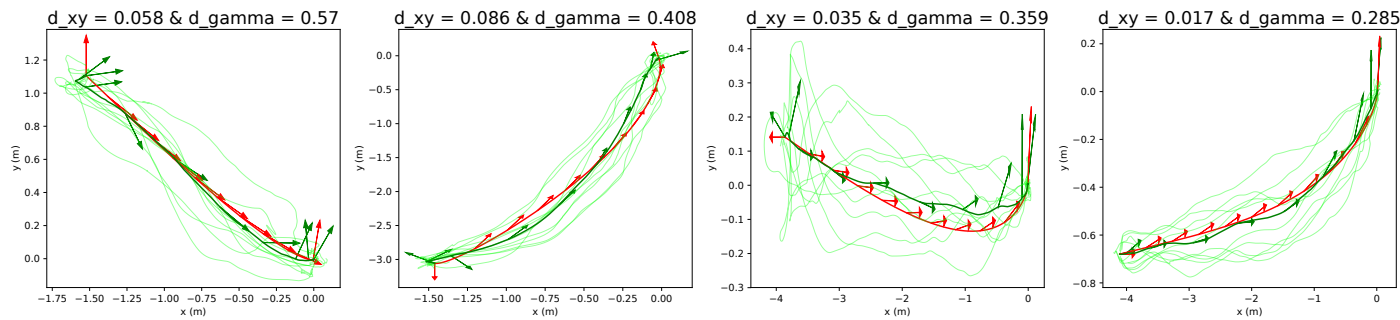

Figure 5. Comparison between human trajectories (average in bold green and measurements in lime green) and generated trajectories (in red) for the start $3\left(\theta_{0}=\frac{\pi}{2} \mathrm{rad}\right), 6\left(\theta_{0}=-\frac{\pi}{2} \mathrm{rad}\right), 8\left(\theta_{0}=\pi \mathrm{rad}\right), 9\left(\theta_{0}=0\right.$ $\mathrm{rad})$ from the left to the right. The arrows represent the orientation of the pelvis during locomotion.
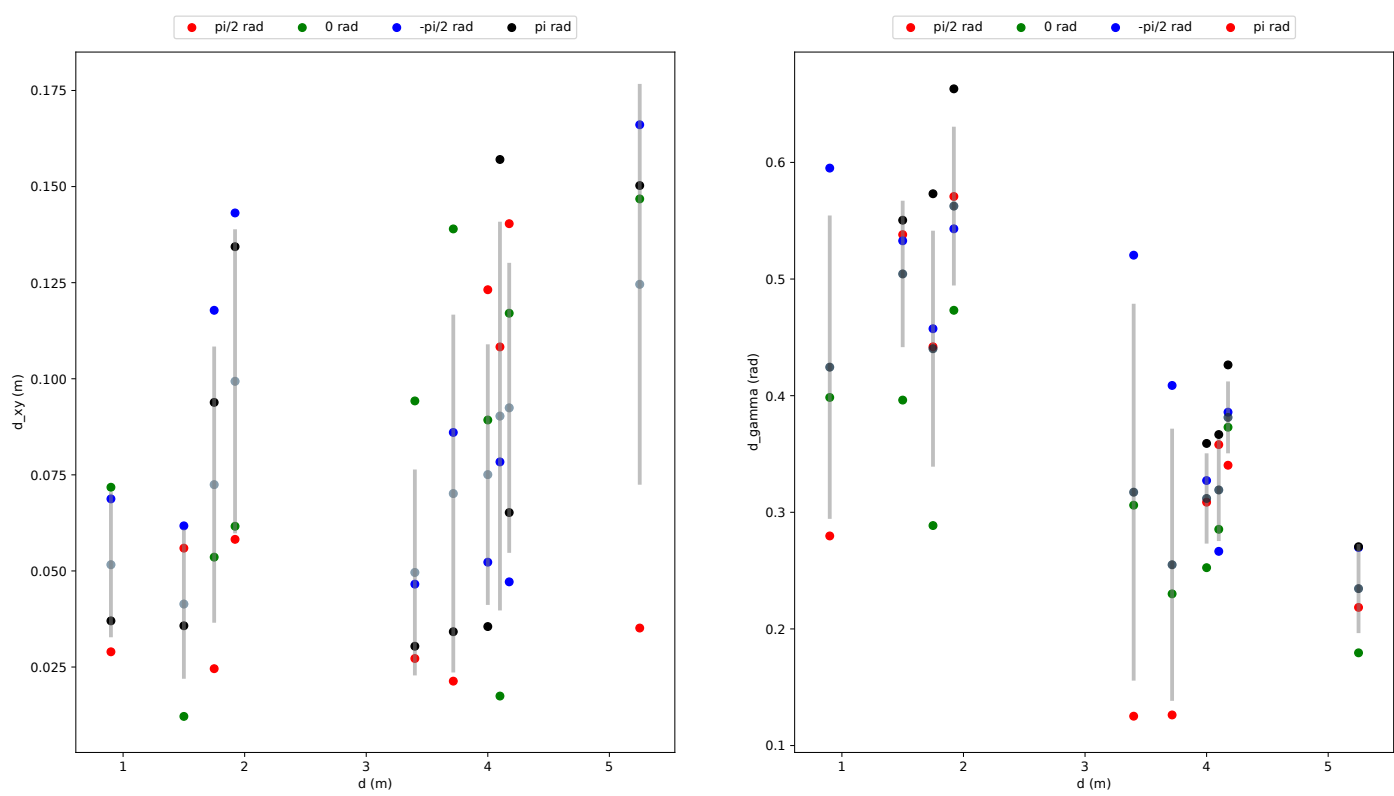

Figure 6. Linear distance (on the left) and angular distance (on the right) between the average human trajectories and the generated trajectories according to the global distance for all orientations (in grey mean \pm standard deviation)

the opposite, when performing a kruskal test with the linear error and the distance between the measured trajectories of the 6 indistinguishable subjects and the average human trajectories, the $\mathrm{p}$-value is superior to the threshold $(p=0.07)$. Thus, it can be asserted that the OC model precisely describes the average human behaviour. Nevertheless, it cannot fit every individual behaviours (6 over 10 in this study).

The box and whisker plots for linear and angular distances are represented on Fig.7a (on the left). Similar plots are presented in Fig.7b where the linear distances are split into 4 groups one for each starting orientations and in Fig. $7 \mathrm{c}$ where they are split into 2 groups according to the global distance superior or inferior to $3 \mathrm{~m}$. As these diagrams present similar medians, they show no significant differences between those groups. ANOVA tests confirmed this assumption. Indeed, the p-value was superior to the threshold when applying the test to the 4 orientation-discriminated groups $\left(p_{x y}=0.68\right)$, to the 2 global distance-discriminated groups $\left(p_{x y}=0.24\right)$ and to every groups $\left(p_{x y}=0.71\right)$. This demonstrates that the presented model provides 
homogeneous results for every global distance (within the chosen range) and every orientation (among the chosen ones).

All the presented results are reproducible as all the libraries are open-source and the source code and the data are available on: https://github.com/imaroger/walking_ human_trajectory_models.

\section{Discussion}

First, the present work proposes a study of human walking trajectories. The CoM trajectories in the horizontal plane and posterio-anterior orientations of the pelvis of 10 subjects walking freely from one start to a goal were collected. The analysis of the measured trajectories shows a great variability between the subjects. This paper also implements a new OC model solved with DDP to simulate human-like trajectories without taking into account the medio-lateral oscillations. This model was optimized using the measured trajectories through an IOC scheme. The assessment of the model shows mean linear and angular errors around, respectively, $0.08 \mathrm{~m}$ and $0.38 \mathrm{rad}$. Those results are of the same order of magnitude than the mean distances between the average human trajectories and the measured trajectories. Thus, this model provides a close approximation of the average human CoM trajectory and pelvis orientation during locomotion. Moreover, its computational time allows real-time application on a humanoid robot which was not the case in Maroger et al. (2020b). However, it is still too slow to fluidly assist a human due to the present limits of humanoid robot. Further works on the TALOS walking pattern generator may push those limits to make the robot reach a more human-like walking velocity.

In this paper, the weights of the cost function (Eq4) were optimized. A quick analysis of this result shows that the weight factor corresponding to the angular acceleration is very low, close to zero. This means that either the orientation of the pelvis does not influence human walking trajectories, which does not seem reasonable, or, more certainly, this factor is redundant with the forward and orthogonal accelerations and the $\psi$ function which all depend on $\theta$. On the opposite, the weight associated to the orthogonal acceleration is quite important in regard to the the weight of the forward acceleration. This demonstrates the importance of the possibility for sideward motions which is totally missing in non-holonomic models, like the unicycle model. This corroborates the starting hypothesis to consider a model with additional degrees of freedom rather than a non-holonomic model to best fit human locomotion. Thus, a holonomic system is an accurate approximation of a human subject. Those weights can be compared to the ones computed in Mombaur et al. (2010). They are not of the same magnitude especially for the weight factor corresponding to the angular acceleration. The reason why those weights are so different remains unclear but it may be due to the difference of solver and of the experimental setup as the distances performed in their experiment did not exceed $3.5 \mathrm{~m}$.

To the best of our knowledge, few authors compare a human-like locomotion path to measurements using a metrics similar to the one presented in this paper. Even in Mombaur et al. (2010), there is no numerical assessment of the distance between the human trajectories and those generated with the proposed model. So in this paper, we go further introducing a metrics to endorse our results. However, it is difficult to 

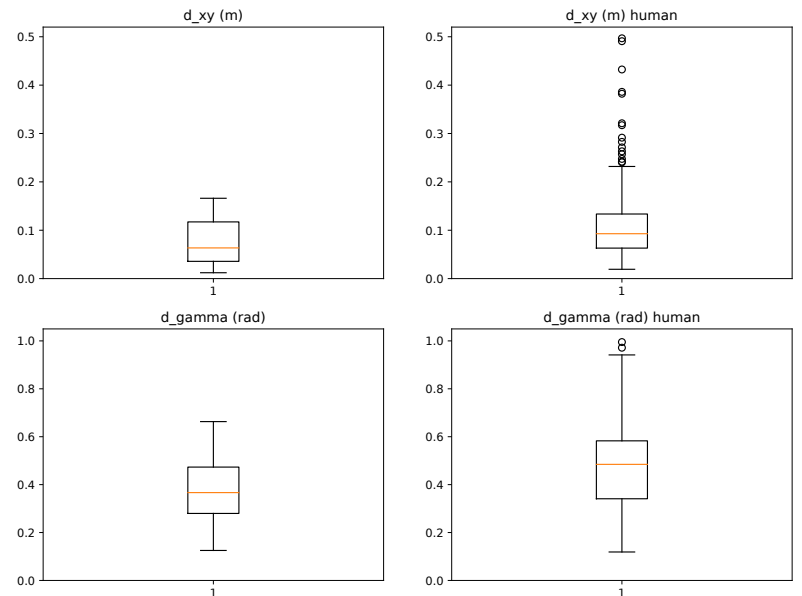

(a) Linear and angular distance distribution: OC model on the left and human measurements on the right.

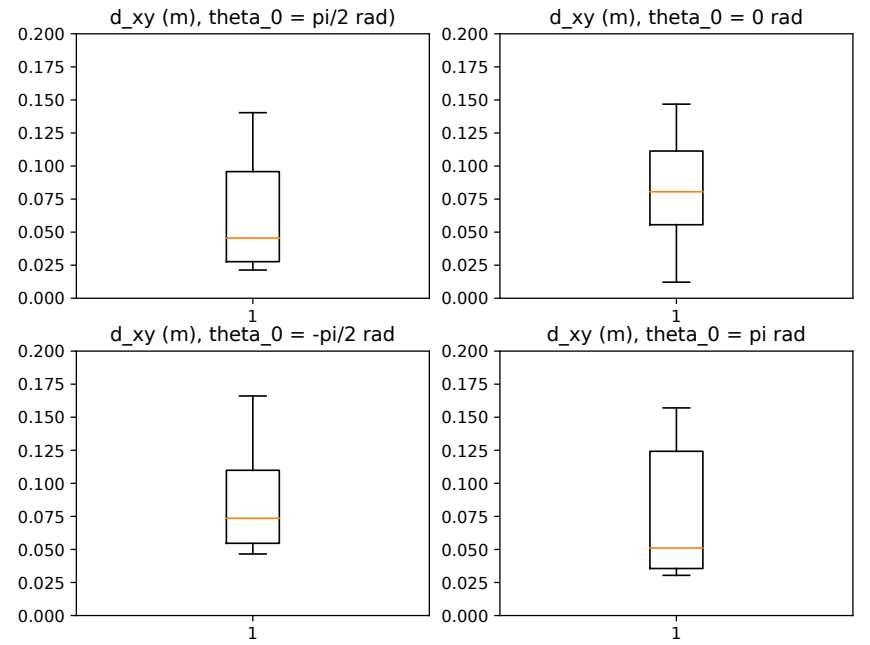

(b) Linear distance distribution of generated trajectories according to the starting orientation $\left(\theta_{0}\right)$.
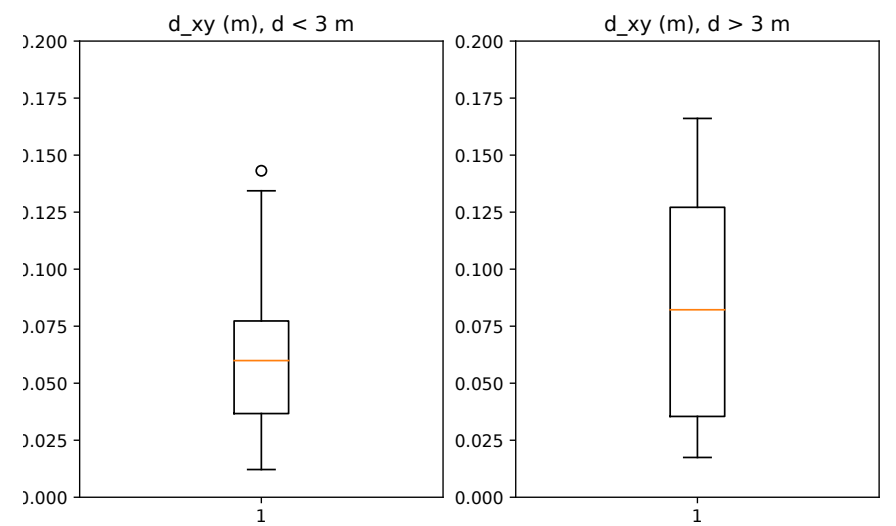

(c) Linear distance distribution of generated trajectories according to the global distance $(d)$.

Figure 7. Distribution of the computed distance 
assess the presented results with respect to other studies. Only in Arechavaleta et al. (2008), a similar metrics is used to compute the linear error and alike results are found with a model based on the unicycle model. However, not a lot of information are given about their results. So, a comparison with our model only leads to the conclusion that we have results of the same order of magnitude for the linear error. There is no evaluation of the angular error in the literature as far as we know. At least, the results presented in this paper can be compared with the results of our previous works (Maroger et al. 2020b a). In those studies, the authors implemented an OC model similar to the one introduced in this paper using the cost function weights proposed by Mombaur et al. (2010). Comparison shows that the OC model described in this article is much better than the ones tested in those former studies where the mean linear distance was around $0.2 \mathrm{~m}$ and the mean angular distance around $0.9 \mathrm{rad}$ using the same metrics.

Even if our study leads to a model which correctly approximates human behaviour, it has some limitations. First of all, the presented model only has got a weak optimality constraint on the final state. This can lead not to exactly reach the goal position. However, for the generated trajectories, the average final distances are $0.016 \mathrm{~m}$ and $0.19 \mathrm{rad}$ which is largely acceptable for the robotic targeted application namely a collaborative task between a humanoid robot and a human.

Furthermore, the orientation computation is flawed. Indeed, during the measurements some markers were obliterated and data was not collected. We were unable to compute the orientation of the subjects'pelvis for 125 over 400 analysed trajectories. This leads to 3 over 40 average human trajectories without average orientations. These trajectories were not taken into account in the IOC process and in the distance computation.

Moreover, the presented OC model greatly depends on the chosen dynamics (Eq1) and the chosen cost function for the OC (Eq, 4) and for the IOC (Eq,8). Indeed, other choices can be made for the OC cost function and maybe adding or removing terms can improve the model. For example further studies may analyse the velocity profile in order to also make it fit to human dynamics and introduce a term which binds the velocity to the curvature of the trajectory to generate realistic velocity profiles. Thus, the velocity profile may respect the two-thirds power law (Viviani and Flash 1995). Jerk (Flash and Hogan 1985) or kinetic energy (Biess et al.|2007) terms could also be added to the cost function. However, in (Mombaur et al. 2010), the authors show that adding velocity and jerk terms to the cost function does not improve their model. Moreover, terms can be added to the cost function for the IOC problem like a distance evaluating the closeness between the linear and angular velocity profiles. Nevertheless, weights must be added as the different distances will not be on the same scale and a manual search of these weights may be a long process as each IOC run takes at least 36 hours.

This leads to another potential weakness of our method: the IOC scheme is very computation time consuming. This prevents the customisation of the model according to each subject which could improve the generated trajectories. Indeed, we showed that the trajectories taken by the subjects present a great variability. This is why it may be relevant to individually recompute the $\mathrm{OC}$ cost function weights $\alpha$ and $\beta$ for each subject. However, the huge computation time of the IOC scheme does not allow this 
re-computation for real-time application like collaborative tasks. Nevertheless, using another method to solve the IOC problem might speed up the weights computation. The Powell method used in this work might be replaced by a genetic algorithm (Sylla et al. 2014) or an Inverse Karush-Kuhn-Tucker (KKT) approach (Panchea et al. 2018) or a probabilistic method like Inverse Reinforcement Learning (IRL) (Park and Levine 2013). The implementation of these methods are more complex but they may be more efficient and faster to solve an IOC problem.

\section{Conclusion}

In this paper, a study of human trajectories during locomotion is presented. A reproducible OC model which generates smooth human-like CoM trajectories between a starting position and a goal position is proposed. The trajectories generated with this model well fit human behaviour for every distance between the starting and the goal positions and for every starting orientations according to the defined metrics. The targeted applications of this work are, for now, to allow a humanoid robot to walk to a table in a human-like way and, in future works, to swiftly follow or even anticipate human walking trajectories while it assists a human to carry and move a table. In this context, the results obtained in this paper are reasonable as the error between the average human trajectories and the generated trajectories is around $0.08 \mathrm{~m}$ which means that the OC model fits the average human behaviour. This model may allow to achieve the ANR-COBOT project's goals in future works.

\section{Acknowledgements}

The authors would like to thank the participants who volunteered for the experiment conducted in this study as well as H. Pillet and N.A. Turpin for their useful comments to improve the manuscript.

\section{Disclosure statement}

The authors declare no known conflict of interest, financial or otherwise, that could bias their work.

\section{Funding}

This work was funded by the French National Research Agency (ANR) as part of the project ANR-COBOT [18CE10-0003]. Study sponsor did not have a role in the study design, the data collection, analysis and interpretation, the writing of the manuscript and the decision to submit the manuscript for publication.

\section{References}

Arechavaleta G, Laumond J, Hicheur H, Berthoz A. 2008. An optimality principle governing human walking. IEEE Transactions on Robotics. 24(1):5-14. 
Arechavaleta G, Laumond JP, Hicheur H, Berthoz A. 2001. The nonholonomic nature of human locomotion: A modeling study. In: Proceeding of the IEEE/RAS-EMBS International Conference on Biomedical Robots and Biomechatronics; vol. 2006; 02. p. 158 - 163.

Arechavaleta G, Laumond JP, Hicheur H, Berthoz A. 2008. On the nonholonomic nature of human locomotion. Autonomous Robots. 25:25-35.

Basili P, Saglam M, Kruse T, Huber M, Kirsch A, Glasauer S. 2013. Strategies of locomotor collision avoidance. Gait posture. 37 3:385-90.

Biess A, Liebermann D, Flash T. 2007. A computational model for redundant human threedimensional pointing movements: Integration of independent spatial and temporal motor plans simplifies movement dynamics. The Journal of Neuroscience. 27:13045 - 13064.

Bovi G, Rabuffetti M, Mazzoleni P, Ferrarin M. 2011. A multiple-task gait analysis approach: kinematic, kinetic and emg reference data for healthy young and adult subjects. Gait Posture. 33(1):6-13.

Elbanhawi M, Simic M, Jazar R. 2015. Continuous path smoothing for car-like robots using b-spline curves. In: Journal of Intelligent Robotic Systems; vol. 80; 01.

Flash T, Hogan N. 1985. The coordination of arm movements: an experimentally confirmed mathematical model. In: The Journal of neuroscience : the official journal of the Society for Neuroscience.

Gard C, Miff C, Kuo A. 2004. Comparison of kinematic and kinetic methods for computing the vertical motion of the body center of mass during walking. In: Human movement science. p. $597-610$.

Heijnen M, Muir BC, Rietdyk S. 2012. Factors leading to obstacle contact during adaptive locomotion. Experimental Brain Research. 223:219-231.

Kim J, Bertram JEA. 2018. Compliant walking appears metabolically advantageous at extreme step lengths. Gait posture. 64:84-89.

Koilias A, Nelson MG, Anagnostopoulos CN, Mousas C. 2020. Immersive walking in a virtual crowd: The effects of the density, speed, and direction of a virtual crowd on human movement behavior. Computer Animation and Virtual Worlds. 31(6):e1928.

Kosuge K, Yoshida H, Fukuda T. 1993. Dynamic control for robot-human collaboration. In: Proceedings of 1993 2nd IEEE International Workshop on Robot and Human Communication. p. 398-401.

Laumond JP. 1998. Robot motionplanning and control. In: Lectures Notes in Control and Information Sciences 229, Springer.

Maroger I, Stasse O, Watier B. 2020a. Comparison of human experimental trajectories and simulations during gait. In: Computer Methods in Biomechanics and Biomedical Engineering (in press).

Maroger I, Stasse O, Watier B. 2020b. Walking human trajectory models and their application to humanoid robot locomotion. In: IEEE/RSJ International Conference on Intelligent Robots and Systems (in press).

Mastalli C, Budhiraja R, Merkt W, Saurel G, Hammoud B, Naveau M, et al. 2020. Crocoddyl: An efficient and versatile framework for multi-contact optimal control. In: IEEE International Conference on Robotics and Automation.

Mombaur K, Laumond JP, Truong A. 2009. An inverse optimal control approach to human motion modeling. In: Springer Tracts in Advanced Robotics; vol. 70. p. 451-468.

Mombaur K, Truong A, Laumond JP. 2010. From human to humanoid locomotion - an inverse optimal control approach. Autonomous Robots:369-383.

Nandi GC, Semwal VB, Raj M, Jindal A. 2016. Modeling bipedal locomotion trajectories using hybrid automata. In: 2016 IEEE Region 10 Conference (TENCON). p. 1013-1018.

Panchea A, Ramdani N, Bonnet V, Fraisse P. 2018. Human arm motion analysis based on the inverse optimization approach. 08. p. 1005-1010.

Papadopoulos A, Bascetta L, Ferretti G. 2013. Generation of human walking paths. In: IEEE International Conference on Intelligent Robots and Systems; 11.

Park T, Levine S. 2013. Inverse optimal control for humanoid locomotion.

Powell MJD. 1964. An efficient method for finding the minimum of a function of several 
variables without calculating derivatives. The Computer Journal. 7(2):155-162.

Raković M, Savić S, Santos-Victor J, Nikolić M, Borovac B. 2019. Human-inspired online path planning and biped walking realization in unknown environment. Frontiers in Neurorobotics. $13: 36$.

Semwal VB, Kumar C, Mishra PK, Nandi GC. 2018. Design of vector field for different subphases of gait and regeneration of gait pattern. IEEE Transactions on Automation Science and Engineering. 15(1):104-110.

Sheridan TB. 2016. Human-robot interaction: Status and challenges. Human Factors. 58(4):525-532.

Soueres P, Laumond J. 1996. Shortest paths synthesis for a car-like robot. IEEE Transactions on Automatic Control. 41(5):672-688.

Stasse O, et al. 2017. Talos: A new humanoid research platform targeted for industrial applications. In: IEEE-RAS International Conference on Humanoid Robotics. p. 689-695.

Sylla N, Bonnet V, Venture G, Armande N, Fraisse P. 2014. Human arm optimal motion analysis in industrial screwing task. In: 5th IEEE RAS/EMBS International Conference on Biomedical Robotics and Biomechatronics. p. 964-969.

Tassa Y, Mansard N, Todorov E. 2014. Control-limited differential dynamic programming. In: IEEE International Conference on Robotics and Automation.

Virtanen P, Gommers R, Oliphant TE, Haberland M, Reddy T, Cournapeau D, Burovski, et al. 2020. SciPy 1.0: Fundamental Algorithms for Scientific Computing in Python. Nature Methods. 17:261-272.

Viviani P, Flash T. 1995. Minimum-jerk, two-thirds power law, and isochrony: converging approaches to movement planning. Journal of experimental psychology Human perception and performance. 21(1):32-53.

Zhu Y, Ren D, Fan M, Qian D, Li X, Xia H. 2020. Robust trajectory forecasting for multiple intelligent agents in dynamic scene. CoRR. abs/2005.13133. 